\title{
Central Configurations in the Charged Three Body Problem
}

\author{
Ernesto Perez-Chavela, Antoni Susin, and Zhiming Yan \\ . In this paper we find the exact number and type of Central Config- \\ urations in the general charged three body problem for any set of parameters \\ (masses and charges). Because of the change of the forces, the number of Cen- \\ tral Configurations will be different from the general Newtonian three body \\ problem. Analyzing all the possible changes of sign of the forces, we obtain \\ the different numbers of Central Configurations.
}

\section{Introduction}

The Newtonian $n$-body problem concerns the motion of $n$ point particles with masses $m_{j} \in R^{+}$and positions $\vec{r}_{j} \in R^{3}(j=1, \ldots, n)$, when moving under the Newtonian law of attraction. The equations of motion are

$$
m_{j} \ddot{\vec{r}}_{j}=\sum_{i \neq j} \frac{m_{i} m_{j}\left(\vec{r}_{i}-\vec{r}_{j}\right)}{r_{i j}^{3}}=\frac{\partial U}{\partial \vec{r}_{j}},
$$

where $r_{i j}=\left|\vec{r}_{i}-\vec{r}_{j}\right|$, and the potential $U$ is given by

$$
U=\sum_{i<j} \frac{m_{i} m_{j}}{r_{i j}}
$$

By definition

$$
\Delta_{i j}=\left\{\left(\vec{r}_{1}, \ldots, \vec{r}_{n}\right) \in R^{3 n} \quad \mid \quad \vec{r}_{i}=\vec{r}_{j}\right\}
$$

and

$$
\Delta=\bigcup_{i<j} \Delta_{i j}
$$

the configuration space is

$$
X=R^{3 n} \backslash \Delta .
$$

Definition 1.1. A point $r_{0} \in X$ is a central configuration $(C C)$, if there is some scalar $\alpha$ so that $\alpha r_{0}=\nabla U\left(r_{0}\right)$.

1991 Mathematics Subject Classification. Primary 58F05; Secondary $70 H 99$.

Research partially supported by CONACYT grant 1772-E9210. Research of the second author is also supported by CIRIT grant BE94/2-219 and UPC grant PR9409. These results were first obtained by Z. Yan $[Y]$ in his PhD thesis with D. Saari as thesis advisor. Later and independently, the other two authors derived similar conclusions. Therefore, we decided to combine our efforts in one publication. 
Defining the matrix $\bar{M}=\operatorname{diag}\left(m_{1}, m_{1}, m_{1}, \ldots, m_{n}, m_{n}, m_{n}\right)$ we find that $r_{0}$ is a $C C$ iff $\nabla U\left(r_{0}\right)=\lambda \bar{M} r_{0}$ where $\lambda=U\left(r_{0}\right) / r_{0}^{t} \bar{M} r_{0}$. Then the study of the central configurations is equivalent to the analysis of the last equation, which can be seen as a problem of Lagrange multipliers, where $r_{0}^{t} \bar{M} r_{0}=1$ is a constraint, so if we define

$$
\Omega=\left\{r \in R^{3 n} \backslash \Delta \mid m_{1} r_{1}+\ldots+m_{n} r_{n}=0, r^{t} \bar{M} r=1\right\},
$$

then $r_{0}$ is a $C C$ iff it is a critical point of the restriction of $U(r)$ to $\Omega$.

For the three body problem, there are five central configurations (or just four if we accept rotations in $R^{3}$ ); which corresponds to three collinear central configurations (Euler's configurations), and two equilateral triangle central configurations (Lagrange's configurations). For all choices of masses $m_{1}, m_{2}, m_{3}$, the last two $C C$ always corresponds to non-degenerate minimun of the potential $U$ restricted to $\Omega$, so they are stable. Not as much is known about central configurations for the $n^{-}$ body problem when $n>3$. Even it is not clear whether the number of $C C$ is finite or not.

In this paper we will study the central configurations in the charged three body problem. Here, analyzing the potential $U$ we can see that, depending on the parameters, the forces can have different signs. Then the possible number of $C C$ could change from the general Newtonian three body problem. To get the different numbers of $C C$, we introduce new parameters, in terms of the original ones, which permits to count the exact number of $C C$ for any set of parameters.

\section{Equations for the Central Configurations}

Consider three point particles with masses $m_{1}, m_{2}, m_{3} \in R^{+}$and charges $q_{1}, q_{2}, q_{3} \in R$. Let $\overrightarrow{r_{i}}$ be the position vector of the particle $i$, and fix the origin at the mass center of the system. The potential $U$, and the momentum of inertia $I$ are given by

$$
\begin{gathered}
U=\frac{m_{1} m_{2}-q_{1} q_{2}}{r_{12}}+\frac{m_{2} m_{3}-q_{2} q_{3}}{r_{23}}+\frac{m_{1} m_{3}-q_{1} q_{3}}{r_{13}}=\frac{\lambda_{3}}{r_{12}}+\frac{\lambda_{1}}{r_{23}}+\frac{\lambda_{2}}{r_{13}}, \\
I=m_{1} r_{1}^{2}+m_{2} r_{2}^{2}+m_{3} r_{3}^{2}=\frac{1}{M} \sum_{i<j} m_{i} m_{j} r_{i j}^{2} .
\end{gathered}
$$

Where $r_{i j}=\left|\overrightarrow{r_{i}}-\overrightarrow{r_{j}}\right|,\left|\overrightarrow{r_{i}}\right|=r_{i}, M=m_{1}+m_{2}+m_{3}, \lambda_{i}=m_{j} m_{k}-q_{j} q_{k}$ where $(i, j, k)$ permutes cyclically in $(1,2,3)$, and units of time have been chosen to set the gravitational constant $G=1$.

As in the Newtonian three body problem, $\vec{r} \in\left(R^{3}\right)^{3}$ is a $C C$ iff

$$
\nabla\left(I U^{2}\right)=0 \text { at } \vec{r}
$$

Since the center of mass is fixed at the origin, we can introduce Jacobi's coordinates to reduce the number of independent variables.

Let

$$
\begin{aligned}
& \vec{s}_{1}=\vec{r}_{2}-\vec{r}_{1}, \\
& \vec{s}_{2}=\vec{r}_{3}-\frac{m_{1} \vec{r}_{1}+m_{2} \vec{r}_{2}}{m_{1}+m_{2}} .
\end{aligned}
$$

Using above relationship between $\vec{s}_{1}, \vec{s}_{2}$ and $\vec{r}_{1}, \vec{r}_{2}, \vec{r}_{3}$ and the fact that the center of mass is fixed at the origin, that is $m_{1} \vec{r}_{1}+m_{2} \vec{r}_{2}+m_{3} \vec{r}_{3}=0$, we have (see fig. 1) 

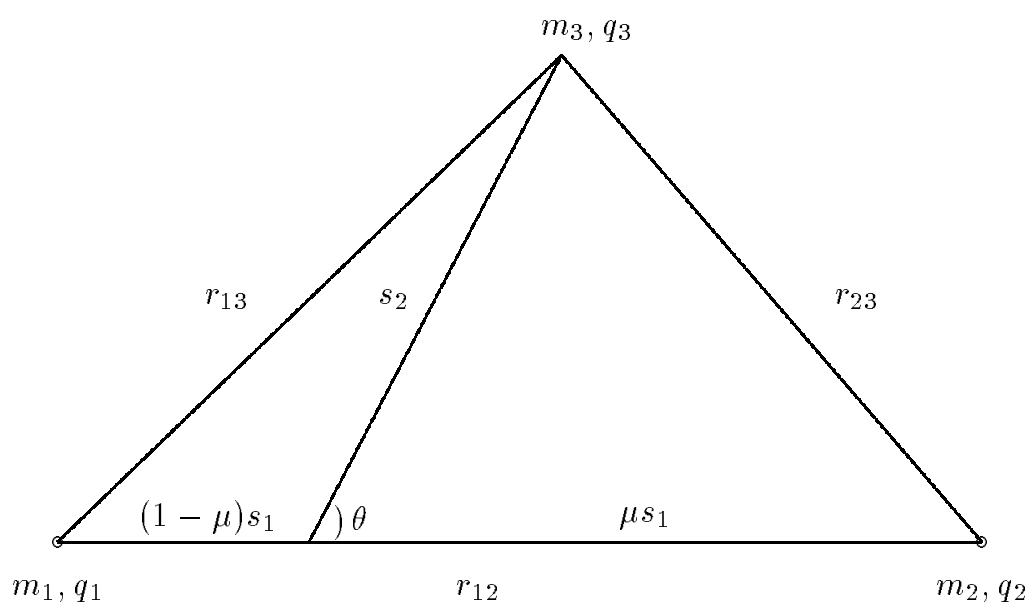

Figure 1. The Jacobi Coordinate System $J_{12}$

$$
\begin{aligned}
\vec{r}_{1} & =-\frac{m_{2}}{m_{1}+m_{2}} \vec{s}_{1}-\frac{m_{3}}{m_{1}+m_{2}+m_{3}} \vec{s}_{2}, \\
\vec{r}_{2} & =\frac{m_{1}}{m_{1}+m_{2}} \vec{s}_{1}-\frac{m_{3}}{m_{1}+m_{2}+m_{3}} \vec{s}_{2}, \\
\vec{r}_{3} & =\frac{m_{1}+m_{2}}{m_{1}+m_{2}+m_{3}} \vec{s}_{2} .
\end{aligned}
$$

Expressing $U$ and $I$ in terms of $\left|\vec{s}_{1}\right|=s_{1}$ and $\left|\vec{s}_{2}\right|=s_{2}$ we have:

$$
\begin{gathered}
U=\frac{\lambda_{3}}{s_{1}}+\frac{\lambda_{1}}{\sqrt{s_{2}^{2}+\left(\mu s_{1}\right)^{2}-2 \mu s_{1} s_{2} \cos \theta}}+\frac{\lambda_{2}}{\sqrt{s_{2}^{2}+\left((1-\mu) s_{1}\right)^{2}+2(1-\mu) s_{1} s_{2} \cos \theta}}, \\
I=g_{1} s_{1}^{2}+g_{2} s_{2}^{2} .
\end{gathered}
$$

Where $\theta$ is the angle between the vectors $\vec{s}_{1}$ and $\vec{s}_{2}$ and

$$
\mu=\frac{m_{1}}{m_{1}+m_{2}}, \quad g_{1}=\frac{m_{1} m_{2}}{m_{1}+m_{2}}, \quad g_{2}=\frac{m_{3}\left(m_{1}+m_{2}\right)}{m_{1}+m_{2}+m_{3}} .
$$

So we have

$$
\frac{1}{g_{2}} I U^{2}=\left(\beta+\rho^{2}\right)\left(\lambda_{3}+\frac{\lambda_{1}}{\sqrt{\rho^{2}+\mu^{2}-2 \mu \rho \cos \theta}}+\frac{\lambda_{2}}{\sqrt{\rho^{2}+(1-\mu)^{2}+2(1-\mu) \rho \cos \theta}}\right)^{2},
$$

where $\beta=g_{1} / g_{2}$ and $\rho=s_{2} / s_{1}$.

Now that $I U^{2}$ is in terms of the parameters $\lambda_{1}, \lambda_{2}, \lambda_{3}, \beta, \mu$ and the two independent variables $\rho$, and $\theta$, the formula for the central configurations of the charged three body problem is

$$
\begin{aligned}
& \frac{\partial}{\partial \rho}\left(\frac{1}{g_{2}} I U^{2}\right)=0, \\
& \frac{\partial}{\partial \theta}\left(\frac{1}{g_{2}} I U^{2}\right)=0 .
\end{aligned}
$$

The fact that $I U^{2}$ remains unchanged under rotation and scalar change of the system requiers the same condition to apply for the central configurations in the charged three body problem. 


\section{Symmetries}

The Jacobi System of the previous section, where the base vector is $\vec{s}_{1}=\vec{r}_{2}-\vec{r}_{1}$, is denoted by $J_{12}$. Denote the selected Jacobi System, where $\vec{s}_{1}=\vec{r}_{3}-\vec{r}_{1}$ is the base vector, and $\vec{s}_{2}$ is the vector which goes from mass center of masses $m_{1}$ and $m_{3}$ to $m_{2}$, as $J_{13}$. The remain Jacobi System is denoted by $J_{21}$. Because there exists an one to one correspondence between these Jacobi Systems, all of our analysis is in the Jacobi System $J_{12}$. An analysis of the collinear central configurations $(C C C)$ in the Jacobi System $J_{12}$ reduces to whether particle $m_{3}$, is between the other two, or exterior to them.

Let $p_{i}$ be the position on a fixed line of particle $m_{i}$ for $i=1,2,3$. In the next section we prove that it is sufficient to study the case where $p_{1}, p_{2}$ and $p_{3}$ are aligned in this order, that will be called Case I. In the system $J_{12}$ this corresponds to $\theta=0, \rho>\mu$. The other possible $C C C$ correspond to the alignments $p_{3}, p_{1}, p_{2}$ (Case II), and $p_{1}, p_{3}, p_{2}$ (Case III).

For Case II, in system $J_{12}$ we have that $\theta=\pi$ and $\rho>1-\mu$. Which corresponds to the study of the Case I in the system $J_{21}$. To express the potential $U$ in these variables it is necessary to introduce the changes $\bar{m}_{1}=m_{2}, \bar{m}_{2}=m_{1}, \bar{m}_{3}=$ $m_{3}, \bar{q}_{1}=q_{2}, \bar{q}_{2}=q_{1}, \bar{q}_{3}=q_{3}$, and with these changes we have $\bar{\lambda}_{1}=\lambda_{2}, \bar{\lambda}_{2}=$ $\lambda_{1}, \bar{\lambda}_{3}=\lambda_{3}$.

For the Case III, in the System $J_{12}$, we have two possibities

(i) $\theta=0, \quad \rho<\mu$,

(ii) $\theta=\pi, \quad \rho<1-\mu$.

Both possibilities correspond to study the Case I in the system $J_{13}$, where now the respective changes are $\hat{m}_{1}=m_{1}, \hat{m}_{2}=m_{3}, \hat{m}_{3}=m_{2}, \hat{q}_{1}=q_{1}, \hat{q}_{2}=q_{3}, \hat{q}_{3}=$ $q_{2}$, and with these changes we have $\hat{\lambda}_{1}=\lambda_{1}, \hat{\lambda}_{2}=\lambda_{3}, \hat{\lambda}_{3}=\lambda_{2}$.

\section{Noncollinear Central Configurations}

Let $R_{i j}$ be the relative distance between $p_{i}$ and $p_{j}$ with respect to $s_{1}=r_{12}$, i.e.

$$
R_{13}=\frac{r_{13}}{s_{1}}, \quad R_{23}=\frac{r_{23}}{s_{1}}, \quad R_{12}=\frac{r_{12}}{s_{1}}=1 .
$$

So

$$
\frac{1}{g_{2}} I U^{2}=\left(\beta+\rho^{2}\right)\left[\lambda_{3}+\frac{\lambda_{1}}{R_{23}}+\frac{\lambda_{2}}{R_{13}}\right]^{2} .
$$

From system 2.1 central configurations satisfy

$$
\begin{aligned}
& \rho\left(\lambda_{3}+\frac{\lambda_{1}}{R_{23}}+\frac{\lambda_{2}}{R_{13}}\right)+\left(\rho^{2}+\beta\right)\left[-\frac{\lambda_{1}(\rho-\mu \cos \theta)}{R_{23}^{3}}-\frac{\lambda_{2}(\rho+(1-\mu) \cos \theta)}{R_{13}^{3}}\right]=0, \\
& \quad-\frac{\lambda_{1} \rho \mu \sin \theta}{R_{23}^{3}}+\frac{\lambda_{2}(\rho(1-\mu) \sin \theta)}{R_{13}^{3}}=0 .
\end{aligned}
$$

Solving the second equation, we have

$$
\rho \sin \theta=0 \quad \text { or } \quad \frac{m_{2} \lambda_{2}}{R_{13}^{3}}=\frac{m_{1} \lambda_{1}}{R_{23}^{3}} .
$$

In the case of $\rho \sin \theta=0$, we have $\rho=0$ or $\sin \theta=0$ which corresponds to collinear $C C$. We will study this case later in the next section. 
Now consider the second case and let

$$
\frac{m_{2} \lambda_{2}}{R_{13}^{3}}=\frac{m_{1} \lambda_{1}}{R_{23}^{3}}=z^{-3} .
$$

Then we can solve for $R_{13}$ and $R_{23}$ and substitute them into equation 4.2 , obtaining

$$
z^{3}=\frac{1}{m_{3} \lambda_{3}}
$$

So the unique solution is

$$
\frac{m_{1} \lambda_{1}}{R_{23}^{3}}=\frac{m_{2} \lambda_{2}}{R_{13}^{3}}=\frac{m_{3} \lambda_{3}}{R_{12}^{3}}=m_{3} \lambda_{3} .
$$

Only when $\lambda_{1}, \lambda_{2}, \lambda_{3}$, have the same sign does this solution define an unique central configuration. Physically this means that all charges have the same polarity. Using the above relation we can check that this central configuration is a triangle with one body at each vertex if the parameters satisfy the constraint

$$
\left(m_{i} \lambda_{i}\right)^{1 / 3}+\left(m_{j} \lambda_{j}\right)^{1 / 3}>\left(m_{k} \lambda_{k}\right)^{1 / 3}
$$

where $(i, j, k)$ permutes cyclically in $(1,2,3)$. Note that this triangle could be of any shape depending on the choice of the parameters.

We want to remark that if all $\lambda_{i}$ have the same sign and the constraint 4.5 holds, then we can always construct the relation 4.4 , which guaranties the existence of non-collinear $C C$.

Observe that in the Jacobi System $J_{12}$, if the angle $\theta$ is replaced by $-\theta$, we get the same result about the existence of non-collinear $C C$. This just reflets the symmetry of the potential function $U$,

$$
U\left(s_{1}, s_{2}, \theta\right)=U\left(s_{1}, s_{2},-\theta\right)
$$

Because three points in $R^{3}$ define a plane, we only consider rotations in the plane. Therefore the existence of one non-collinear $C C$ implies the existence of the symmetric one, which must be considered different because is not obtained by planar rotation of the original one. One special case is when all charges are zero. Then $m_{1} \lambda_{1}=m_{2} \lambda_{2}=m_{3} \lambda_{3}=m_{1} m_{2} m_{3}$. The solution will be

$$
\frac{1}{R_{23}^{3}}=\frac{1}{R_{13}^{3}}=\frac{1}{R_{12}^{3}}=1,
$$

or

$$
R_{12}=R_{13}=R_{23}=1
$$

let us observe that in this case the constraint 4.5 holds in a trivial way, so here we get the Lagrange's configurations for the classical Newtonian 3-body problem.

If any two of $\lambda_{1}, \lambda_{2}, \lambda_{3}$ have different signs, then the solution does not define any configuration, which means that non-collinear $C C$ does not exist.

In this way, we can determine the total number of non-collinear $C C$ which can be 0 or 2 , according to the values of the parameters (or more precisely, the signs of some parameters must satisfices the conditions 4.4 and 4.5 ). 


\section{Collinear Central Configurations}

In the last section we obtain two possible conditions associated to the existence of collinear central configurations $(C C C)$. Now we will study these two possibilities. (i) $\rho=0$.

The solution for all $\theta$ of equation 4.2 becomes

$$
\frac{\lambda_{1}}{\mu^{2}}=\frac{\lambda_{2}}{(1-\mu)^{2}}
$$

Thus only when the parameters satisfy 5.1 can there be a $C C$ with $m_{3}$ sitting at the center of masses $m_{1}$ and $m_{2}$. This is intuitive because Eq. 5.1 requires the attracting force on $m_{3}$ by $m_{2}$ (repelling force if $\lambda_{1}$ is negative) to equal the attracting force on $m_{3}$ by $m_{1}$, so $m_{3}$ can remains at the mass center forever. This corresponds to case III in our symmetries.

(ii) $\sin \theta=0$

The two possibilities are $\theta=0$ and $\theta=\pi$.

For $\theta=0$, we only need to consider $\rho>\mu$, as $0<\rho<\mu$ corresponds to case III.

Similarly $\theta=\pi$, causes $0<\rho<1-\mu$ or $\rho>1-\mu$ which correspond to case III and case II respectively.

Now we start with the case $\theta=0, \quad \rho>\mu$ (case I), when $\lambda_{i} \neq 0, i=1,2,3$. Using $r_{23}=s_{2}-\mu s_{1}$, Eq. 4.2 becomes

$$
F(\rho)=\lambda_{3} \rho-\frac{\lambda_{1} \mu\left(\rho+\frac{\beta}{\mu}\right)}{(\rho-\mu)^{2}}+\frac{\lambda_{2}(1-\mu)\left(\rho+\frac{\beta}{1-\mu}\right)}{(\rho+1-\mu)^{2}}=0 .
$$

To simplify this function, we introduce new parameters. Let

$$
\begin{aligned}
& \alpha_{1}=m_{1} \lambda_{1}, \\
& \alpha_{2}=m_{2} \lambda_{2}, \\
& \alpha_{3}=\left(m_{1}+m_{2}\right) \lambda_{3},
\end{aligned}
$$

and

$$
\begin{aligned}
& \beta_{1}=\frac{\alpha_{1}}{\alpha_{2}}, \\
& \beta_{3}=\frac{\alpha_{3}}{\alpha_{2}} .
\end{aligned}
$$

Also let $x=\rho-\mu$ so in this case, $x>0$.

With these new parameters, we have

$$
F(\rho)=\frac{f(x) \alpha_{2}}{m_{1}+m_{2}}
$$

where

$$
f(x)=\beta_{3} x+\beta_{3} \mu-\frac{\beta_{1}\left(x+1+\frac{m_{2}}{m_{3}}\right)}{x^{2}}+\frac{x-\frac{m_{1}}{m_{3}}}{(x+1)^{2}} .
$$

By choosing $m_{3}$ as the unit of mass, we can let $m_{3}=1$. So the above equation becomes

$$
f(x)=\beta_{3} f_{3}(x)-\beta_{1} f_{1}(x)+f_{2}(x)=0,
$$

where

$$
\begin{gathered}
f_{3}(x)=x+\frac{m_{1}}{m_{1}+m_{2}}>0 \\
f_{1}(x)=\frac{x+1+m_{2}}{x^{2}}>0
\end{gathered}
$$




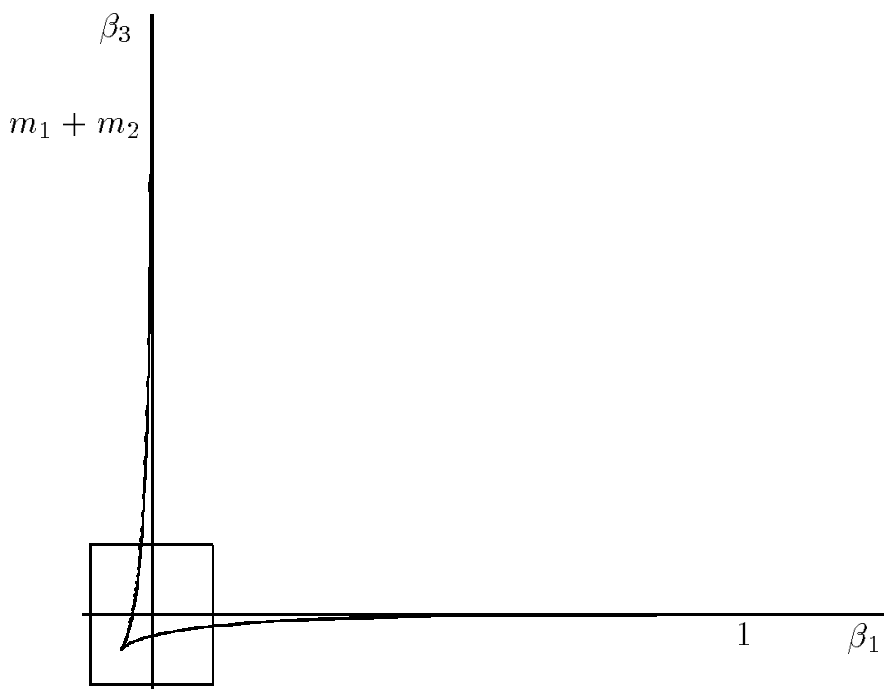

Figure 2. The Bifurcation Curve for $m_{1}=m_{2}=m_{3}=1$.

From 5.4 we have

$$
f_{2}(x)=\frac{x-m_{1}}{(x+1)^{2}}
$$

$$
\beta_{3}=\beta_{1} g_{1}(x)+g_{2}(x)
$$

where

$$
\begin{gathered}
g_{1}(x)=\frac{f_{1}(x)}{f_{3}(x)}, \\
g_{2}(x)=-\frac{f_{2}(x)}{f_{3}(x)} .
\end{gathered}
$$

In the $\beta_{1} \beta_{3}$ plane Eq. 5.5 becomes a line for a fixed $x$ and fixed masses. Here $g_{1}(x)$ is the slope and $g_{2}(x)$ is the $\beta_{3}$-intercept. For any fixed point $\left(\beta_{1}, \beta_{3}\right)$, the number of lines passing through this point when $x$ is moving from zero to infinity, will determine the number of $C C C$ for that set of parameters.

We now examine how the line changes as $x$ goes from 0 to $+\infty$. First of all,

$$
g_{1}^{\prime}(x)=-\frac{2 x^{2}+\left[3\left(m_{2}+1\right)+\frac{m_{1}}{m_{1}+m_{2}}\right] x+\frac{2 m_{1}\left(m_{2}+1\right)}{m_{1}+m_{2}}}{x^{3}\left(x+\frac{m_{1}}{m_{1}+m_{2}}\right)^{2}}<0,
$$

and $g_{1}(0)=+\infty, g_{1}(+\infty)=0$. So the slope of the line monotonely decreases from $+\infty$ to 0 as $x$ goes from 0 to $+\infty$.

Secondly,

$$
g_{2}^{\prime}(x)=-\frac{-2 x^{2}+\left(3 m_{1}-\frac{m_{1}}{m_{1}+m_{2}}\right) x+\frac{m_{1}\left(3 m_{1}+m_{2}\right)}{m_{1}+m_{2}}+\frac{m_{1}}{m_{1}+m_{2}}}{(x+1)^{3}\left(x+\frac{m_{1}}{m_{1}+m_{2}}\right)^{2}},
$$

and $g_{2}(0)=m_{1}+m_{2}, \quad g_{2}(+\infty)=0$. So we will have

$$
\left\{\begin{array}{l}
g_{2}^{\prime}(x)<0 \text { when } 0<x<x_{+}, \\
g_{2}^{\prime}(x)>0 \text { when } x_{+}<x,
\end{array}\right.
$$


where

$$
x_{+}=\frac{\left(3 m_{1}-\frac{m_{1}}{m_{1}+m_{2}}\right)+\sqrt{\left(3 m_{1}-\frac{m_{1}}{m_{1}+m_{2}}\right)^{2}+8\left(\frac{m_{1}\left(3 m_{1}+m_{2}\right)}{m_{1}+m_{2}}+\frac{m_{1}}{m_{1}+m_{2}}\right)}}{4} .
$$

So the $\beta_{3}$-intercept of the line decreases from $m_{1}+m_{2}$ when $0<x<x_{+}$and then increases to 0 when $x>x_{+}$.

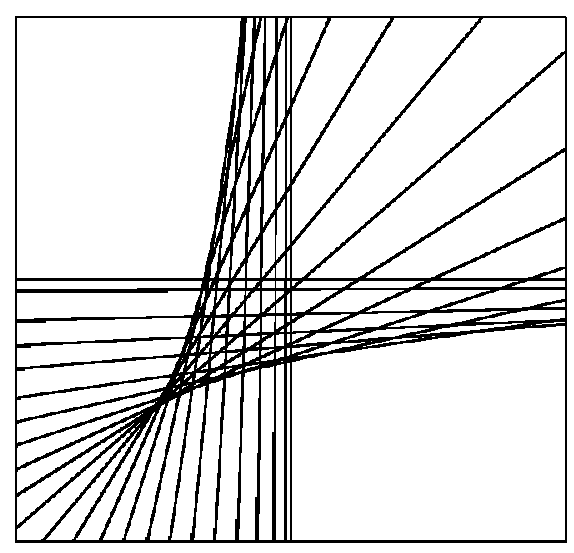

Figure 3. Detail of the family of lines associated to the Bifurcation Curve for $m_{1}=m_{2}=m_{3}=1$.

Based on the analysis of $g_{1}(x)$ and $g_{2}(x)$, we can sketch in the $\beta_{1} \beta_{3}$ plane the changes of the line as $x$ goes from 0 to $+\infty$. Thus we get some regions in the $\beta_{1} \beta_{3}$ plane and in each region the number of times the line passes through any point in that region is the same, i.e. the number of $C C C$ remains fixed (see Fig.3).

We can also get the bifurcation curve between regions computing the envelope of the family of lines given by the equation 5.5. For this, we derivate 5.5 with respect to $x$, obtaining the parametric form of the bifurcation curve

$$
\begin{aligned}
& \beta_{1}(x)=-\frac{g_{2}^{\prime}(x)}{g_{1}^{\prime}(x)} \\
& \beta_{3}(x)=-\frac{g_{2}^{\prime}(x)}{g_{1}^{\prime}(x)} g_{1}(x)+g_{2}(x) .
\end{aligned}
$$

which is a piecewise smooth curve. It is easy to check that $\lim _{x \rightarrow \infty} \beta_{1}(x)=1$ and $\lim _{x \rightarrow \infty} \beta_{3}(x)=0$. The bifurcation curve given by 5.6, and the coordinate axis in the $\beta_{1} \beta_{3}$ plane determine the regions where we get the same number of $C C C$, this number could be $0,1,2$ or 3 (see fig. 4 ).

\section{Number of Collinear Central Configurations}

In order to get the total number of $C C C$ we need to work with different Jacobi systems. To use the bifurcation curve (Eq. 5.6) obtained in the above section when changing 


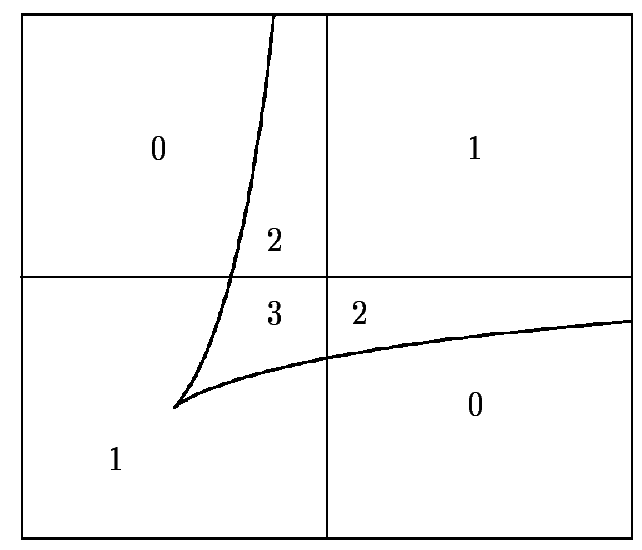

FigurE 4. Different regions according to the number of $C C C$.

the Jacobi system we need that all the parameters $\lambda_{i} \neq 0, \quad i=1,2,3$. In order to point out the relation between two different Jacobi systems, we introduce the rate charge-mass $\delta_{i}=\frac{q_{i}}{m_{i}}, i=1,2,3$. So

$$
\lambda_{i}=m_{j} m_{k}\left(1-\delta_{j} \delta_{k}\right) \text { where }(i, j, k) \text { permutes cyclically in }(1,2,3) .
$$

With this notation

$$
\beta_{1}=\frac{1-\delta_{2} \delta_{3}}{1-\delta_{1} \delta_{3}}, \quad \beta_{3}=\left(\frac{m_{1}+m_{2}}{m_{3}}\right) \frac{1-\delta_{1} \delta_{2}}{1-\delta_{1} \delta_{3}} .
$$

We denote by $\bar{\beta}_{1}$ and $\bar{\beta}_{3}$ the analogous variables in the Jacobi system $J_{21}$ (case II), and by $\hat{\beta}_{1}$ and $\hat{\beta}_{3}$ the respective ones in the Jacobi system $J_{13}$ (case III). By the symmetries given in section 3 , we get

$$
\begin{array}{ll}
\bar{\beta}_{1}=\frac{1}{\beta_{1}}, & \hat{\beta}_{1}=\left(\frac{m_{1}+m_{2}}{m_{3}}\right) \frac{\beta_{1}}{\beta_{3}}, \\
\bar{\beta}_{3}=\frac{\beta_{3}}{\beta_{1}}, & \hat{\beta}_{3}=\frac{\left(m_{1}+m_{2}\right)\left(m_{1}+m_{3}\right)}{m_{2} m_{3}} \frac{1}{\beta_{3}} .
\end{array}
$$

Let us observe that in order to get the $\bar{\beta}_{\mathrm{s}}$ and $\hat{\beta}_{\mathrm{s}}$ we need $\lambda_{i} \neq 0$ for $i=1,2,3$. Therefore we split the study of the number of $C C C$ in two subsections depending on $\lambda_{1} \lambda_{2} \lambda_{3} \neq 0$ or not.

6.1. All the $\lambda$ 's do not vanish. In the above section we have studied the case $\theta=0$ and $\rho>\mu$ in the Jacobi system $J_{12}$. Using the symmetries given in section 3 , we will obtain the total number of $C C C$ according to the different signs of the parameters $\lambda_{i}$.

We will denote by $1 Q, 2 Q, 3 Q$ and $4 Q$ the first, second, third and fourth quadrants respectively, corresponding to the plane $\beta_{1} \beta_{3}$ associated to the Jacobi system $J_{12}$. Analogously we use the same notation with $\left(^{-}\right)$or $\left(^{{ }^{\wedge}}\right)$ for the Jacobi systems $J_{21}$ and $J_{13}$. And we will also denote by $B C, \bar{B} \bar{C}, \hat{B} \hat{C}$ to the bifurcation curve in the respective Jacobi system. 
(i) $\lambda_{1}>0, \lambda_{2}>0, \lambda_{3}>0$ (resp. $\left.\lambda_{1}<0, \lambda_{2}<0, \lambda_{3}<0\right)$.

In this case we have $\beta_{1}>0$ and $\beta_{3}>0$, then according to the bifurcation diagram given in the above section, we only have one $C C C$, because the point $\left(\beta_{1}, \beta_{3}\right) \in 1 Q$ in the Jacobi system $J_{12}$. Now using the relations given by 6.2 and 6.3 the points $\left(\bar{\beta}_{1}, \bar{\beta}_{3}\right) \in 1 \bar{Q}$ and $\left(\hat{\beta}_{1}, \hat{\beta}_{3}\right) \in 1 \hat{Q}$ (the first quadrants of the respective bifurcation diagrams).

Therefore, in this case we have exactely $3 C C C$.

(ii) $\lambda_{1}>0, \lambda_{2}<0, \lambda_{3}<0$ (resp. $\lambda_{1}<0, \lambda_{2}>0, \lambda_{3}>0$ ).

In this case we have :

$\left(\beta_{1}, \beta_{3}\right) \in 2 Q \backslash B C$, so we can have 0 or $2 C C C$, or 1 if $\left(\beta_{1}, \beta_{3}\right) \in B C$. $\left(\bar{\beta}_{1}, \bar{\beta}_{3}\right) \in 3 \bar{Q} \backslash \bar{B} \bar{C}$, so we can have 1 or $3 C C C$, or 2 if $\left(\bar{\beta}_{1}, \bar{\beta}_{3}\right) \in \bar{B} \bar{C}$. $\left(\hat{\beta}_{1}, \hat{\beta}_{3}\right) \in 2 \hat{Q} \backslash \hat{B} \hat{C}$, so we can have 0 or $2 C C C$ or 1 if $\left(\hat{\beta}_{1}, \hat{\beta}_{3}\right) \in \hat{B} \hat{C}$.

Therefore, in this case we can have $1,2,3,4,5,6$ or $7 C C C$.

(iii) $\lambda_{1}<0, \lambda_{2}>0, \lambda_{3}<0$ (resp. $\lambda_{1}>0, \lambda_{2}<0, \lambda_{3}>0$ ).

In this case we have :

$\left(\beta_{1}, \beta_{3}\right) \in 3 Q \backslash B C$, so we can have 1 or $3 C C C$, or 2 if $\left(\beta_{1}, \beta_{3}\right) \in B C$. $\left(\bar{\beta}_{1}, \bar{\beta}_{3}\right) \in 2 \bar{Q} \backslash \bar{B} \bar{C}$, so we can have 0 or $2 C C C$, or 1 if $\left(\bar{\beta}_{1}, \bar{\beta}_{3}\right) \in \bar{B} \bar{C}$. $\left(\hat{\beta}_{1}, \hat{\beta}_{3}\right) \in 4 \hat{Q} \backslash \hat{B} \hat{C}$, so we can have 0 or $2 C C C$, or 1 if $\left(\hat{\beta}_{1}, \hat{\beta}_{3}\right) \in \hat{B} \hat{C}$.

Therefore, in this case we can have $1,2,3,4,5,6$ or $7 C C C$.

(iv) $\lambda_{1}<0, \lambda_{2}<0, \lambda_{3}>0$ (resp. $\lambda_{1}>0, \lambda_{2}>0, \lambda_{3}<0$ ).

In this case we have :

$\left(\beta_{1}, \beta_{3}\right) \in 4 Q \backslash B C$, so we can have 0 or $2 C C C$, or 1 if $\left(\beta_{1}, \beta_{3}\right) \in B C$.

$\left(\bar{\beta}_{1}, \bar{\beta}_{3}\right) \in 4 \bar{Q} \backslash \bar{B} \bar{C}$, so we can have 0 or $2 C C C$ or 1 if $\left(\bar{\beta}_{1}, \bar{\beta}_{3}\right) \in \bar{B} \bar{C}$.

$\left(\hat{\beta}_{1}, \hat{\beta}_{3}\right) \in 3 \hat{Q} \backslash \hat{B} \hat{C}$, so we can have 1 or $3 C C C$ or 2 if $\left(\hat{\beta}_{1}, \hat{\beta}_{3}\right) \in \hat{B} \hat{C}$.

Therefore, in this case we can have $1,2,3,4,5,6$ or $7 C C C$.

Now we will analyze if all these $C C C$ are possible. From 5.3 we have

$\beta_{3} x^{3}(x+1)^{2}+\beta_{3} \mu x^{2}(x+1)^{2}-\beta_{1}\left(x+1+\frac{m_{2}}{m_{3}}\right)(x+1)^{2}+\left(x-\frac{m_{1}}{m_{3}}\right) x^{2}=0$.

This polynomial can be written as

$$
p(x)=a_{5} x^{5}+a_{4} x^{4}+a_{3} x^{3}+a_{2} x^{2}+a_{1} x+a_{0}=0,
$$

where

$$
\begin{aligned}
& a_{5}=\beta_{3}, \\
& a_{4}=\beta_{3}(2+\mu) \\
& a_{3}=\beta_{3}(1+2 \mu)-\beta_{1}+1 \\
& a_{2}=\beta_{3} \mu-\beta_{1}\left(3+\frac{m_{2}}{m_{3}}\right)-\frac{m_{1}}{m_{3}} \\
& a_{1}=-\beta_{1}\left(3+2 \frac{m_{2}}{m_{3}}\right) \\
& a_{0}=-\beta_{1}\left(1+\frac{m_{2}}{m_{3}}\right) .
\end{aligned}
$$


The $C C C$ correspond to the positive roots of 6.5 , in this form we can prove in a different way the above statements about the number of possible $C C C$. Now we will prove that is impossible to have the cases with 6 or $7 C C C$.

We start analyzing the case

(ii) $\lambda_{1}>0, \lambda_{2}<0, \lambda_{3}<0$ (resp. $\lambda_{1}<0, \lambda_{2}>0, \lambda_{3}>0$ ).

The idea is to prove that $\left(\beta_{1}, \beta_{3}\right) \in 2 Q \backslash B C$ and $\left(\hat{\beta}_{1}, \hat{\beta}_{3}\right) \in 2 \hat{Q} \backslash \hat{B} \hat{C}$ can not be simultaneously in the region of $2 C C C$.

Let us observe that in this case $a_{5}, a_{4}, a_{3}, a_{1}$ and $a_{0}$ are positive, and the same is true for the $\hat{a}_{i}, i=5,4,3,1,0$, so in order to have $\left(\beta_{1}, \beta_{3}\right)$ and $\left(\hat{\beta}_{1}, \hat{\beta}_{3}\right)$ in the same region of $2 C C C$ is necessary by the Descartes' rule of signs to find the positive roots of $p(x)$ that, $a_{2}<0$ and $\hat{a}_{2}<0$, that is

$$
\begin{aligned}
& \beta_{3} \mu-\beta_{1}\left(3+\frac{m_{2}}{m_{3}}\right)-\frac{m_{1}}{m_{3}}<0, \\
& \hat{\beta}_{3} \hat{\mu}-\hat{\beta}_{1}\left(3+\frac{\hat{m}_{2}}{\hat{m}_{3}}\right)-\frac{\hat{m}_{1}}{\hat{m}_{3}}<0 .
\end{aligned}
$$

Now using the symmetries studied in the part 3 of this paper we can write the above equations in terms of $\beta_{1}, \beta_{3}, \mu, m_{1}, m_{2}$, and $m_{3}$, so

$$
\begin{gathered}
\beta_{3} \mu-\beta_{1}\left(3+\frac{m_{2}}{m_{3}}\right)-\frac{m_{1}}{m_{3}}<0, \\
\frac{m_{1}\left(m_{1}+m_{2}\right)}{m_{2} m_{3}}-\beta_{1}\left(3+\frac{m_{3}}{m_{2}}\right)\left(\frac{m_{1}+m_{2}}{m_{3}}\right)-\frac{m_{1}}{m_{2}} \beta_{3}<0 .
\end{gathered}
$$

Multipying the first equation 6.6 by $\frac{m_{1}}{m_{2}}$, the second by $\mu$ and adding we get

$$
-\beta_{1}\left[\left(3+\frac{m_{2}}{m_{3}}\right) \frac{m_{1}}{m_{2}}+\left(\frac{m_{1}+m_{2}}{m_{3}}\right)\left(3+\frac{m_{3}}{m_{2}}\right) \mu\right]<0,
$$

which is a contradiction. The analysis in the cases $i i i)$ and $i v$ ) is similar. Therefore we have proved the following result.

Proposition 6.1. If all the $\lambda_{i}$ 's do not vanish, is possible to have 1, 2, 3, 4 or $5 C C C$.

6.2. Some $\lambda_{i}$ vanish. Different possibilities. Now we will study the nongeneric cases, that is when $\lambda_{i}=0$ for some $i=1,2,3$, and therefore, we can not use the bifurcation diagram given in section 5 . Moreover, we can not have non-collinear $C C$. We will start our study with the simplest cases.

(i) $\lambda_{1}=\lambda_{2}=\lambda_{3}=0$.

This is a totally degenerate situation. Here the potential function $U \equiv 0$ therefore we do not have any $C C C$.

(ii) $\lambda_{1}=\lambda_{2}=0, \lambda_{3} \neq 0$.

Here the potential $U$ takes the form $U=\frac{\lambda_{3}}{r_{12}}$. Thus, the charged three body problem is reduced to a Kepler problem, attractive or repulsive, depending on the sign of $\lambda_{3}$, and it is well known that there is not any $C C C$. The other two cases when only one $\lambda_{i} \neq 0$ give the same result.

(iii) $\lambda_{1}=0, \lambda_{2} \neq 0, \lambda_{3} \neq 0$. 
When only one $\lambda_{i}$ vanishes we will use the same symmetries given in section 3 . Therefore, it is enough to study the case when $\theta=0$ and $\rho>\mu$.

¿From the equation 5.2 we have

$$
F(\rho)=\lambda_{3} \rho+\frac{\lambda_{2}(1-\mu)\left(\rho+\frac{\beta}{1-\mu}\right)}{(\rho+1-\mu)^{2}}=0 .
$$

Let $x=\rho-\mu>0$. Then the above equation takes the form

$$
\lambda_{2}=G(x) \lambda_{3}
$$

where

$$
G(x)=-\frac{(x+\mu)(x+1)^{2}}{(x+\mu)(1-\mu)+\beta} .
$$

Let us observe that

$-1<G(0)=-\frac{\mu}{\mu(1-\mu)+\beta}<0, \quad \lim _{x \rightarrow+\infty} G(x)=-\infty \quad$ and $\quad G^{\prime}(x)<0 \quad \forall x>0$.

Therefore, when $x$ goes from 0 to $+\infty$, we have the following bifurcation diagram (see fig. 5). As a consequence we will have 0 or $1 \mathrm{CCC}$.

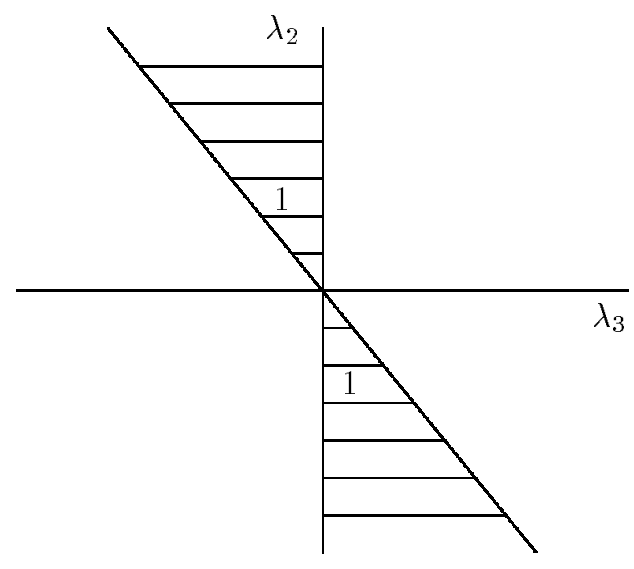

Figure 5. Bifurcation diagram for $\lambda_{1}=0, \lambda_{2} \neq 0, \lambda_{3} \neq 0$.

(iv) $\lambda_{1} \neq 0, \lambda_{2}=0, \lambda_{3} \neq 0$.

Like in case (iii), from the equation 5.2 we have

$$
F(\rho)=\lambda_{3} \rho-\frac{\lambda_{1} \mu\left(\rho+\frac{\beta}{\mu}\right)}{(\rho-\mu)^{2}}=0,
$$

and doing $x=\rho-\mu>0$ we have

$$
\lambda_{1}=G(x) \lambda_{3},
$$

where now

$$
G(x)=\frac{(x+\mu) x^{2}}{\mu(x+\mu)+\beta},
$$


here

$$
G(0)=0, \quad \lim _{x \rightarrow+\infty} G(x)=+\infty, \quad \text { and } \quad G^{\prime}(x)>0 \quad \forall x>0 .
$$

So the bifurcation diagram is given in Figure 6 . Here we will also have 0 or $1 \mathrm{CCC}$.

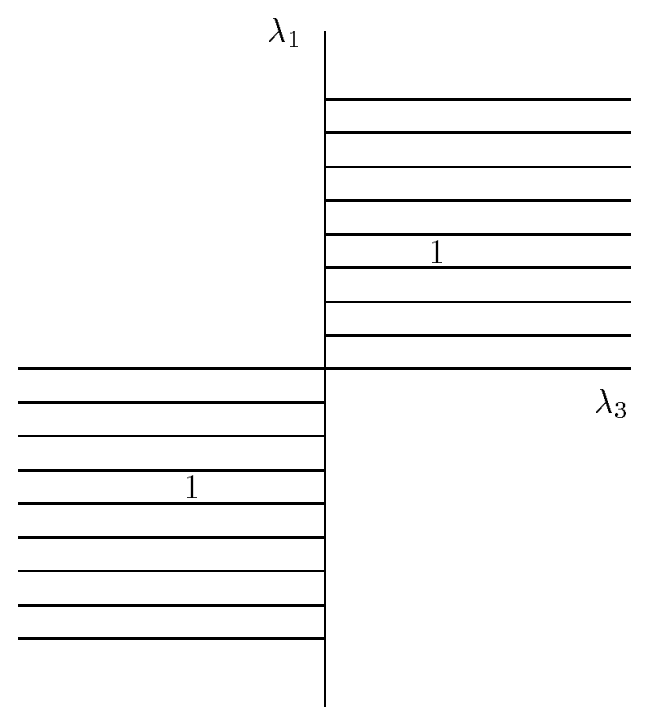

Figure 6. Bifurcation diagram for $\lambda_{1} \neq 0, \lambda_{2}=0, \lambda_{3} \neq 0$.

(v) $\lambda_{1} \neq 0, \lambda_{2} \neq 0, \lambda_{3}=0$.

As we will show, this is the more difficult case. From the equation 5.2, we have

$$
F(\rho)=\frac{\lambda_{2}(1-\mu)\left(\rho+\frac{\beta}{1-\mu}\right)}{(\rho+1-\mu)^{2}}-\frac{\lambda_{1} \mu\left(\rho+\frac{\beta}{\mu}\right)}{(\rho-\mu)^{2}}=0,
$$

and in terms of $x=\rho-\mu>0$ we have

$$
\lambda_{1}=G(x) \lambda_{2}
$$

where now

$$
G(x)=\frac{x^{2}[(x+\mu)(1-\mu)+\beta]}{(x+1)^{2}[\mu(x+\mu)+\beta]}
$$

Let us observe that

$$
G(0)=0, \quad \lim _{x \rightarrow+\infty} G(x)=\frac{1-\mu}{\mu}, \quad \text { and } \quad G(x)<1 \quad \forall x>0 .
$$

The sign of the derivative of $G(x)$ is given by the sign of its numerator which is the polynomial

$$
q(x)=x p(x)=x\left(a x^{3}+b x^{2}+c x+d\right),
$$

where

$$
\begin{aligned}
& a=\beta(1-2 \mu)+2 \mu(1-\mu) \\
& b=2(1-\mu)\left(2 \beta+\mu+2 \mu^{2}\right) \\
& c=3(1-\mu)\left(\beta+\mu^{2}\right)+(\beta+\mu(1-\mu))\left(2 \beta+\mu+2 \mu^{2}\right) \\
& d=2\left(\mu^{2}+\beta\right)(\beta+\mu(1-\mu))
\end{aligned}
$$


We point out that the coefficients $b, c$ and $d$ are always positive for any choice of the parameters, while the coefficient $a$ can have any sign. Writing $a$ in terms of the mass parameters, we obtain

$$
a=\frac{m_{1} m_{2}}{\left(m_{1}+m_{2}\right)^{2}}\left[\frac{M}{m_{3}}\left(\frac{m_{2}-m_{1}}{m_{1}+m_{2}}\right)+2\right],
$$

and then $a \geq 0$ iff $0<\mu \leq \frac{m_{3}}{M}+\frac{1}{2} \equiv \mu_{0}$. In this case $G^{\prime}(x)>0 \quad \forall x>0$. Therefore the bifurcation diagram is shown in Figure 7 . So we can have 0 or $1 C C C$.

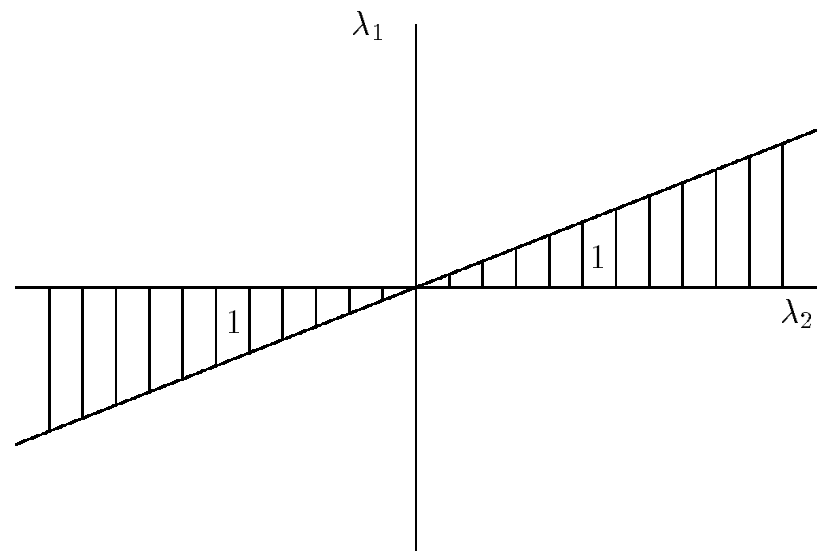

Figure 7. Bifurcation diagram for $\lambda_{1} \neq 0, \lambda_{2} \neq 0, \lambda_{3}=$ 0 and $0<\mu \leq \mu_{0}$.

When $a<0$, that is $\mu_{0}<\mu<1$, by a straightforward but tedious computation, and using the limit behaviour of $G(x)$ at 0 and $+\infty$, we get that $p(x)$ has an unique positive root $x_{0}$. So $G(x)$ is a monotone increasing function in $\left(0, x_{0}\right)$, and then monotonely decreases until $\frac{1-\mu}{\mu}$ in the interval $\left(x_{0},+\infty\right)$.

So the bifurcation diagram is shown in Figure 8 . We point out that now we can have 0,1 or $2 C C C$.

6.3. Some $\lambda_{i}$ 's vanish. Number of collinear central configurations. In section 3 we have seen that

$$
\bar{\lambda}_{1}=\lambda_{2}, \bar{\lambda}_{2}=\lambda_{1} \text { and } \bar{\lambda}_{3}=\lambda_{3},
$$

and also that

$$
\hat{\lambda}_{1}=\lambda_{1}, \hat{\lambda}_{2}=\lambda_{3} \text { and } \hat{\lambda}_{3}=\lambda_{2} \text {. }
$$

In order to count all the $C C C$ we divide our analysis in several cases. By the symmetries of the respective bifurcation diagrams with respect to the origen, in any case is enough to analyze the first two quadrants.

(1.a) $\lambda_{1}=0, \lambda_{2}>0, \lambda_{3}>0$.

$\left(\lambda_{3}, \lambda_{2}\right) \in 1 Q$ so we always have $0 C C C$.

$\left(\bar{\lambda}_{3}, \bar{\lambda}_{1}\right) \in 1 \bar{Q}$ so we always have $1 C C C$.

$\left(\hat{\lambda}_{3}, \hat{\lambda}_{2}\right) \in 1 \hat{Q}$ so we always have $0 C C C$.

Therefore, the only possible number of $C C C$ is 1 . 


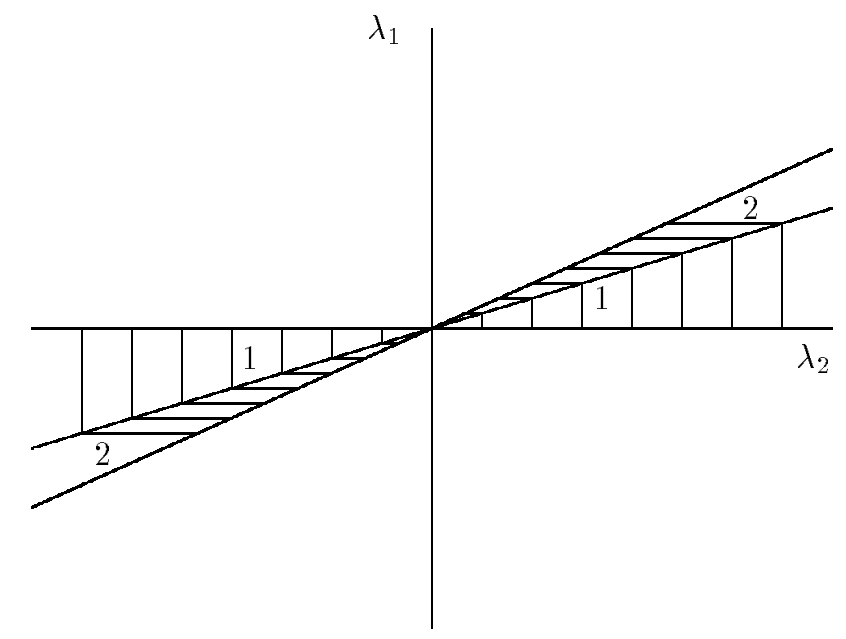

Figure 8. Bifurcation diagram for $\lambda_{1} \neq 0, \lambda_{2} \neq 0, \lambda_{3}=$ 0 and $\mu_{0}<\mu<1$.

(1.b) $\lambda_{1}=0, \lambda_{2}>0, \lambda_{3}<0$.

$\left(\lambda_{3}, \lambda_{2}\right) \in 2 Q$ so we can have 0 or $1 C C C$.

$\left(\bar{\lambda}_{3}, \bar{\lambda}_{1}\right) \in 2 \bar{Q}$ so we always have $0 C C C$.

$\left(\hat{\lambda}_{3}, \hat{\lambda}_{2}\right) \in 4 \hat{Q}$ so we can have 0 or $1 C C C$.

Therefore, the possible number of $C C C$ is 0,1 or 2 .

(2.a) $\lambda_{1}>0, \lambda_{2}=0, \lambda_{3}>0$.

$\left(\lambda_{3}, \lambda_{1}\right) \in 1 Q$ so we always have $1 C C C$.

$\left(\bar{\lambda}_{3}, \bar{\lambda}_{2}\right) \in 1 \bar{Q}$ so we always have $0 C C C$.

$\left(\hat{\lambda}_{2}, \hat{\lambda}_{1}\right) \in 1 \hat{Q}$ so we can have 0 or $1 C C C$ if $0<\mu \leq \mu_{0}$ or we can have 0,1 or $2 C C C$ if $\mu_{0}<\mu<1$.

Therefore, the possible number of $C C C$ is 1,2 or 3 .

(2.b) $\lambda_{1}>0, \lambda_{2}=0, \lambda_{3}<0$.

$\left(\lambda_{3}, \lambda_{1}\right) \in 2 Q$ so we always have $0 C C C$.

$\left(\bar{\lambda}_{3}, \bar{\lambda}_{2}\right) \in 2 \bar{Q}$ so we can have 0 or $1 C C C$.

$\left(\hat{\lambda}_{2}, \hat{\lambda}_{1}\right) \in 2 \hat{Q}$ so we always have $0 C C C$.

Therefore, the possible number of $C C C$ is 0 or 1 .

(3.a) $\lambda_{1}>0, \lambda_{2}>0, \lambda_{3}=0$.

Here we have two possible bifurcation diagrams depending on $\mu \in\left(0, \mu_{0}\right)$ or $\mu \epsilon$ $\left(\mu_{0}, 1\right)$. Let us observe that when we change $J_{12}$ to $J_{21}$, the parameter $\bar{\mu}$ corresponds to $1-\mu$, so is possible that we have to change the respective bifurcation diagrams.

(i) $0<\mu \leq \mu_{0}$.

$\left(\lambda_{2}, \lambda_{1}\right) \in 1 Q$ so we can have 0 or $1 C C C$. 
$\left(\bar{\lambda}_{2}, \bar{\lambda}_{1}\right) \in 1 \bar{Q}$ so we can have 0,1 or $2 C C C$ if the previous one is 0 ; or $0 C C C$ if the previous one is 1 .

$\left(\hat{\lambda}_{3}, \hat{\lambda}_{1}\right) \in 1 \hat{Q}$ so we always have $1 C C C$.

Therefore, the possible number of $C C C$ is 1,2 or 3 .

(ii) $\mu_{0}<\mu<1$.

$\left(\lambda_{2}, \lambda_{1}\right) \in 1 Q$ so we can have 0,1 or $2 C C C$.

$\left(\bar{\lambda}_{2}, \bar{\lambda}_{1}\right) \in 1 \bar{Q}$ so we can have 0 or $1 C C C$ if the previous one is 0 ; or $0 C C C$ if the previous one is 1 or 2 .

$\left(\hat{\lambda}_{3}, \hat{\lambda}_{1}\right) \in 1 \hat{Q}$ so we always have $1 C C C$.

Therefore, the possible number of $C C C$ is 1,2 or 3 .

(3.b) $\lambda_{1}>0, \lambda_{2}<0, \lambda_{3}=0$.

In this case it is not necessary to distinguish the different possibilities for the value of $\mu$, because we always work in the second or fourth quadrants.

$\left(\lambda_{2}, \lambda_{1}\right) \in 2 Q$ so we always have $0 C C C$.

$\left(\bar{\lambda}_{2}, \bar{\lambda}_{1}\right) \in 4 \bar{Q}$ so we always have $0 C C C$.

$\left(\hat{\lambda}_{3}, \hat{\lambda}_{1}\right) \in 2 \hat{Q}$ so we always have $0 C C C$.

Therefore here we do not have any $C C C$.

\section{Total number of Central Configurations}

After our previous analysis, now we can count all the possible central configurations $(C C)$ obtaining the following result.

Theorem 7.1. Given $m_{1}, m_{2}, m_{3} \in R^{+}$and $q_{1}, q_{2}, q_{3} \in R$ we can construct the respective $\lambda_{i}, i=1,2,3$. So

(i) If at least two $\lambda_{i}$ vanish, we have $0 \mathrm{CC}$.

(ii) If just one $\lambda_{i}$ vanishes, then we can have 0,1,2 or $3 C C$.

(iii) If all the $\lambda_{i}$ have the same sign, and $\left(m_{i} \lambda_{i}\right)^{1 / 3}+\left(m_{j} \lambda_{j}\right)^{1 / 3}>\left(m_{k} \lambda_{k}\right)^{1 / 3}$ then we have exactely 5 CC (2 non-collinear and 3 collinear), in the other cases we only have the three collinear $C C$.

(iv) If the $\lambda_{i}$ 's do not vanish and do not have the same sign, then we can have 1, 2, 3, 4 or $5 C C$ (all of them are collinear).

REMARK 7.2. The limit case when the inequality in iii) is an equality, we have a $C C C$, in this case because all the $\lambda_{i}$ have the same sign, we only have the well known three collinear central configurations like in the Newtonian case. So the limit of non-collinear central configurations is a classical collinear central configurations. The big difference between the Newtonian Three Body Problem and the Charged Three Body Problem with respect to central configurations is that in the last case we can have 5 collinear central configurations.

\section{References}

[MH] K. R. Meyer and G. R. Hall, Introduction to Hamiltonian Dynamical Systems and the N-Body Problem, Applied Mathematical Sciences 90, Springer-Verlag (1992).

[M] R. Moeckel, Relative equilibria of the four-body problem, Ergodic Theory Dynamical Systems, 5, (1985), pp. 417-435.

[Mo] R. Moulton, The straight line solutions of the problem of $n$-bodies, Bull. Amer. Math. Soc. 13, (1910), pp. 324-335.

[S] D. G. Saari On the role and properties of $n$-body central configurations, Celestial Mech. 21 (1980), pp. 9-20. 
[X] Z. Xia, Central Configurations with Many Small Masses, Journal of Differential Equations, 91, 1991, pp. 168=179.

[Y] Z. Yan, Restrictions on the charged and regular Newtonian Systems, PhD Thesis, Northwestern University (May 1994).

[W] A. Winter, The Analytical Foundations of Celestial Mechanics, Princeton Univ. Press, Princeton, N.J. 1941.

E-mail address: epc@xanum.uam.mx

E-mail address: susin@ma1.upc.es

E-mail address: z_yan@math.nнu.edu 